\title{
Schwarz lemmas via the pluricomplex Green's function
}

\author{
Jaikrishnan Janardhanan * \\ jaikrishnan@iitm.ac.in \\ Department of Mathematics, Indian Institute of Technology Madras, Chennai 600036, India
}

We prove a version of the Schwarz lemma for holomorphic mappings from the unit disk into the symmetric product of a Riemann surface. Our proof is function-theoretic and self-contained. The main novelty in our proof is the use of the pluricomplex Green's function. We also prove several other Schwarz lemmas using this function.

\section{Introduction}

The main result of this article is the following:

Theorem 1. Let $X$ be a Riemann surface and $f: \mathbb{D} \rightarrow \operatorname{Sym}^{n}(X)$ be holomorphic. Then

$$
\mathcal{H}_{\mathcal{M}_{X}}^{n}\left(\pi_{\text {Sym }}^{-1}(f(x)), \pi_{\text {Sym }}^{-1}\left(f\left(x_{0}\right)\right) \leq \mathcal{M}_{\mathbb{D}}\left(x, x_{0}\right), \quad \forall x, x_{0} \in \mathbb{D} .\right.
$$

The notation used in the theorem will be explained in Section 2 Briefly, $\operatorname{Sym}^{n}(X)$ is the $n$ fold symmetric product of $X$ and $\pi_{\text {Sym }}$ is the natural map from $X^{n}$ to $\operatorname{Sym}^{n}(X)$. The Möbius pseudodistance associated to $X$ is denoted $\mathcal{M}_{X}$ and $\mathcal{H}_{\mathcal{M}_{X}}$ is the Hausdorff pseudodistance induced on subsets of $X$ by $\mathcal{M}_{X}$. Given a point $p \in \operatorname{Sym}^{n}(X), \pi_{\text {Sym }}^{-1}(p)$ here denotes the subset of $X$ comprising the coordinate points of (any element of) $\pi_{\mathrm{Sym}}^{-1}\{p\}$. Note that in our notation, $\pi_{\text {Sym }}^{-1}\{p\}$ is not the same as $\pi_{\text {Sym }}^{-1}(p)$.

Remark 2. Note that Theorem 1 is trivially true whenever $X$ is a compact Riemann surface. Also for a domain $D \subset \mathbb{C}$, it is easy to see that either $D$ is Carathéodory hyperbolic (i.e, the Möbius pseudodistance is a distance) or $\mathcal{M}_{D} \equiv 0$. This is not true for Riemann surfaces; see [Sta75]. We emphasize that Theorem 1 applies to all Riemann surfaces including those for which the Möbius pseudodistance is not a distance but yet not identically 0 .

*Jaikrishnan Janardhanan is supported by a DST-INSPIRE fellowship from the Department of Science and Technology, India.

2010 Mathematics Subject Classification: Primary: 32H15, 32H20, $32 \mathrm{U} 35$

Key words and phrases: Schwarz lemma, symmetric product, pluricomplex Green's function 
The genesis of Theorem 1 is a result by Nokrane and Ransford [NR01, Theorem 1.1] which is in the setting of algebroid multifunction taking values in the unit disk. This was later extended to proper holomorphic correspondences from the unit disk to any bounded planar domain by Chandel [Cha17. Theorem 1.7]. In our notation, the result of Nokrane and Ransford is Theorem 1 with $X=\mathbb{D}$ while that of Chandel is the case when $X$ is any bounded planar domain.

Our motivation for formulating and proving Theorem 1 comes from an earlier work [HJ18] in which we investigated the Minkowski function of a quasi-balanced domain. During the course of our study, we realized that a special case of [NR01, Theorem 1.1] follows easily from simple observations about the Minkowski function and an extremal function (now popularly known as the pluricomplex Green's function) studied by Lempert [Lem81], Klimek [Kli85] and Demailly [Dem87]. A natural question to ask is whether these elementary observations have wider applicability.

The symmetrized bidisk and polydisk have been the subject of intense research for the past two decades; see, for instance, [AY01, EZ05, Nik06, ALY13]. More recently, the symmetric product of more general objects has also been studied by several researchers [CG15. BBDJ18, CG18, Zwo18]. The symmetric product of a Riemann surface can be given a natural complex structure that makes it into a complex manifold. It is, therefore, natural to look for an extension of the original result of Nokrane and Ransford in the setting of symmetric products of a Riemann surface and Theorem 1 is the desired extension. The proofs of both [NR01, Theorem 1.1] and [Cha17, Theorem 1.7] rely on the holomorphic functional calculus (the underlying Banach algebra being the space of $n \times n$ complex matrices). It is unclear how these ideas can be generalized to the setting of an arbitrary Riemann surface. In contrast, our proof of Theorem 1 is almost entirely self-contained and uses tools solely from complex analysis. Specifically, we require only basic facts about plurisubharmonic functions, invariant metrics and some standard theorems from complex analysis. The central tool in our proof is the pluricomplex Green's function alluded to in the previous paragraph.

We will also give several applications that illustrate the scope of our techniques. A case in point is the situation of equality in (1), which can be studied using our techniques in the case when $X=\mathbb{D}$. This has been studied by Nokrane and Ransford [NR01, Theorem 1.2] and our analysis is reminiscent of theirs but simpler.

Theorem 3. Let $f: \mathbb{D} \rightarrow \mathbb{G}^{n}$ be a holomorphic function such that

$$
\mathcal{H}_{\mathcal{M}_{\mathbb{D}}}^{n}\left(\pi^{-1}(f(x)), \pi^{-1}(f(0))=\mathcal{M}_{\mathbb{D}}(x, 0),\right.
$$

for $x \in U$ and $U \subset \mathbb{D}$ a non-empty open subset. Then we can find an automorphism of $\mathbb{D}$, say $g$, such that $g_{\mathrm{Sym}} \circ f$ is the $n$-th root multi-function, i.e., the map

$$
z \mapsto \pi\left(\zeta_{1}(z), \ldots, \zeta_{n}(z)\right)
$$

where $\zeta_{1}(z), \ldots, \zeta_{n}(z) \in \mathbb{D}$ are the $n$-th roots of $z$.

Here $\mathbb{G}^{n}$ is the symmetrized polydisk and $\pi$ is the map whose coordinates are the elementary symmetric polynomials; see Section 2.4 for precise definitions including that of $g_{\text {Sym }}$. 
As another application, we shall also use our techniques to give a Schwarz lemma for quasi-balanced domains that extends the well-known Schwarz lemma for balanced domains (Result 23); see Theorem 25. Using this lemma, we shall then sketch a straightforward proof of a version of Schwarz lemma for the spectral unit ball originally proved by Bharali [Bha07].

\section{Organization}

Section 2 contains a brief treatment of all the tools required in our proofs. We present our Schwarz lemma for quasi-balanced domains in Section 3 . The proofs of Theorems 1 and 3 are contained in Section 4 Finally, we briefly sketch the proof of a version of the Schwarz lemma for the spectral unit ball in Section 5

\section{Notation}

We will use $\mathbb{D}$ to denote the unit disk in the complex plane. The space of holomorphic mappings from a complex manifold $X$ into a complex manifold $Y$ will be denoted $O(X, Y)$. We use $|\cdot|$ for the usual Euclidean norm in $\mathbb{C}^{n}$, irrespective of the dimension. All manifolds will be assumed to be connected. All other notations used will be introduced in Section 2

\section{Acknowledgments}

I would like to thank Dr. Pranav Haridas for many useful discussions. Prof. Aprameyan Parthasarathy and Dr. Vikramjeet Chandel read parts of this manuscript and made useful suggestions that have greatly improved the manuscript. Prof. G.P. Balakumar patiently answered many questions, both trivial and hard, that were useful in the proofs. I would also like to thank Prof. Autumn Kent for bringing Stanton's paper [Sta75] to my attention. I am grateful to the anonymous referee for her/his careful reading of our paper and the many useful suggestions that have improved our exposition.

\section{Tools}

\subsection{The pluricomplex Green's function}

In this section, we define and prove basic facts about an extremal function defined using plurisubharmonic functions. Our treatment is from [Kob98, p. 184] where the definition is attributed to Klimek [Kli85]. The paper by Demailly [Dem87] contains further properties of this function.

Definition 4. Let $X$ be a complex manifold. Fix $z_{0} \in X$ and define the extremal function

$$
\lambda_{X}\left(z, z_{0}\right):=\sup \left\{\phi(z): \phi \in P_{X}\left(z_{0}\right)\right\},
$$

where $P_{X}\left(z_{0}\right)$ is the collection of functions $\phi$ on $X$ that satisfy:

1. $\phi$ is upper semi-continuous, 
2. $0 \leq \phi<1$,

3. $\log \phi$ is plurisubharmonic on $X$,

4. $\phi\left(z_{0}\right)=0$,

5. for any coordinate system $z=\left(z_{1}, \ldots, z_{n}\right)$ with origin at $z_{0}$, the quantity $\frac{\phi(x)}{|z(x)|}$ is bounded above in a neighbourhood of $z_{0}$.

Remark 5. In the above definition, functions that are identically $-\infty$ are considered to be plurisubharmonic whence the function that is identically 0 is an element of $P_{X}\left(z_{0}\right)$. So the collection $P_{X}\left(z_{0}\right)$ is always non-empty.

Remark 6. The function $\log \lambda_{X}\left(z, z_{0}\right)$ is known in the literature as the pluricomplex Green's function with a logarithmic pole at $z_{0}$. The pluricomplex Green's function is well-studied and is at the heart of many deep results (see [Kli91] and the papers cited in the introduction for a small sample). For our purposes, the function $\lambda_{X}$-which we will refer to throughout this paper as the extremal function-is more convenient to work with.

Remark 7. If $D \subset \mathbb{C}^{n}$ is a bounded domain then for each $z_{0} \in D$, the function $\left|z-z_{0}\right| \in P_{D}\left(z_{0}\right)$. Therefore $\lambda_{D}\left(z, z_{0}\right)>0 \forall z \in D \backslash\left\{z_{0}\right\}$.

Lemma 8. Let $X$ and $Y$ be complex manifolds and let $f: X \rightarrow Y$ be holomorphic. Then

$$
\lambda_{Y}\left(f(x), f\left(z_{0}\right)\right) \leq \lambda_{X}\left(x, z_{0}\right) .
$$

Proof. It suffices to show that if $\phi \in P_{Y}\left(f\left(z_{0}\right)\right)$ then $\phi \circ f \in P_{X}\left(z_{0}\right)$. Only the final condition in the definition of $P_{X}\left(z_{0}\right)$ needs to be checked. For a coordinate system $z=\left(z_{1}, \ldots, z_{n}\right)$ around $z_{0}$ and $w=\left(w_{1}, \ldots, w_{n}\right)$ around $f\left(z_{0}\right)$, we have

$$
\begin{aligned}
\log \phi \circ f(x)-\log (|z(x)|) & =\log \phi \circ f(x)-\log |w(f(x))| \\
& +\log \frac{|w(f(x))|}{|z(x)|} .
\end{aligned}
$$

The expression on the right hand side is clearly bounded above in a neighbourhood of $z_{0}$ and we are done.

We need a version of Schwarz lemma for subharmonic functions proved by Sibony in order to compute the extremal function for the unit disk $\mathbb{D}$.

Lemma 9 (Sibony [Sib81] ). Let $u$ be an upper semi-continuous function on $\mathbb{D}$ such that

1. $\log u$ is subharmonic,

2. $\frac{u(z)}{|z|^{2}}$ is bounded on $\mathbb{D}^{*}$,

3. $0 \leq u<1$ on $\mathbb{D}$.

Then $u(z) \leq|z|^{2} \forall z \in \mathbb{D}$. If $u\left(z_{0}\right)=\left|z_{0}\right|^{2}$ for some $z_{0} \in \mathbb{D}, z_{0} \neq 0$, then $u(z) \equiv|z|^{2}$.

Lemma 10. The extremal function $\lambda_{\mathbb{D}}(z, 0)=|z|$.

Proof. Clearly $\lambda_{D}(z, 0) \geq|z|$. Conversely, if $\phi \in P_{\mathbb{D}}(z, 0)$ then $\phi^{2}$ is subharmonic and $\frac{\phi^{2}(z)}{|z|^{2}}$ is bounded above on $\mathbb{D}^{*}$ by the final condition in the definition of $P_{\mathbb{D}}\left(z_{0}\right)$. This means that $\phi(z) \leq|z|$ by Lemma 9 and we are done. 


\subsection{The Möbius pseudodistance}

We now define the Möbius pseudodistance of a complex manifold $X$ and prove some of its key properties.

Definition 11. Let $X$ be a complex manifold. We define the Möbius pseudodistance on $X$ to be

$$
\mathcal{M}_{X}\left(z_{1}, z_{2}\right):=\sup \left\{\left|f\left(z_{1}\right)\right|: f \in O(X, \mathbb{D}), f\left(z_{2}\right)=0\right\} \forall z_{1}, z_{2} \in X .
$$

Remark 12. Observe that the above definition is analogous to that of the Carathéodory pseudodistance except that we use the Möbius distance of $\mathbb{D}$ in the definition instead of the Poincaré distance. The proof that the above definition actually gives a pseudodistance follows along the same lines as that for the Carathéodory pseudodistance. As expected, holomorphic mappings are distance decreasing under this pseudodistance and biholomorphisms are isometries. It is also clear that if $C_{X}$ denotes the Carathéodory pseudodistance on $X$ then $\tanh C_{X}=\mathcal{M}_{X}$. See [JP13, Chapter 2] for details.

Remark 13. It follows from Lemma 10 that

$$
\lambda_{\mathbb{D}}\left(z, z_{0}\right)=\mathcal{M}_{\mathbb{D}}\left(z, z_{0}\right) .
$$

Remark 14. Let $B(a, r)$ be the ball of radius $r$ centred at point $a \in \mathbb{C}^{n}$. Then

$$
\mathcal{M}(z, a)=\frac{|z-a|}{r} \forall z \in B(a, r) .
$$

Remark 15. If $D_{i}$ are disks in the plane then for $\left(z_{1}, \ldots, z_{n}\right) \in D_{1} \times \cdots \times D_{n}$, we have

$$
\mathcal{M}_{D_{1} \times \cdots \times D_{n}}\left(\left(z_{1}, \ldots, z_{n}\right),\left(a_{1}, \ldots, a_{n}\right)\right)=\max _{i} \mathcal{M}_{D_{i}}\left(z_{i}, a_{i}\right) .
$$

Remark 16. Using the Remark 14 and the fact $\mathcal{M}_{X}$ is distance decreasing under the inclusion map, one easily shows that $\mathcal{M}_{X}$ is continuous on $X \times X$; see [JP13. Proposition 2.6.1].

Definition 17. We say that the complex manifold $X$ is Carathéodory hyberbolic if $\mathcal{M}_{X}$ is a distance.

Remark 18. Bounded domains are Carathéodory hyperbolic. This follows from the observation that if $z, w \in D, z \neq w$, then some coordinate projection is a bounded holomorphic function that separates $z$ and $w$.

Lemma 19. Let $X$ be a complex manifold. Then

$$
0 \leq \mathcal{M}_{X}<1 .
$$

Proof. Let $z, w \in X$ be such that $\mathcal{M}_{X}(z, w)=1$. Then by the very definition of $\mathcal{M}_{X}$, we can find a sequence of holomorphic functions $f_{n}: X \rightarrow \mathbb{D}$ such that $f_{n}(w)=0$ and $\left|f_{n}(z)\right| \rightarrow 1$. By Montel's theorem, $O(X, \mathbb{D})$ is a normal family. This means that some subsequence of $f_{n}$ must converge in the compact-open topology to a holomorphic map $f: X \rightarrow \mathbb{D}$. But this is absurd as $\left|f_{n}(z)\right| \rightarrow 1$. 
The next theorem gives the crucial link between the Möbius pseudodistance of a complex manifold and its extremal function. This link is the central tool used in the proof of Theorem 1

Proposition 20. Let $X$ be a complex manifold. Then for a fixed $z_{0} \in X$, the function $\mathcal{M}_{X}\left(\cdot, z_{0}\right)$ is plurisubharmonic. In fact, $\log \mathcal{M}_{X}\left(\cdot, z_{0}\right)$ is plurisubharmonic.

Proof. From Remark $16, \mathcal{M}_{X}$ is a continuous function. The fact that $\mathcal{M}_{X}\left(\cdot, z_{0}\right)$ is plurisubharmonic is now straightforward from the fact that $\mathcal{M}_{X}\left(\cdot, z_{0}\right)$ is continuous and a supremum of plurisubharmonic functions. The same argument also shows that the function $\log \mathcal{M}_{X}\left(\cdot, z_{0}\right)$ is plurisubharmonic.

Remark 21. It is now straightforward to prove that for any $z_{0} \in X$, the function $\mathcal{M}\left(\cdot, z_{0}\right) \in$ $P_{X}\left(z_{0}\right)$. Thus, $\mathcal{M}_{X}\left(\cdot, z_{0}\right) \leq \lambda_{X}\left(\cdot, z_{0}\right)$.

\subsection{The Minkowski function of a quasi-balanced domain}

Let $p_{1}, p_{2}, \ldots, p_{n}$ be relatively prime positive integers. We say that a domain $D \subset \mathbb{C}^{n}$ is $\left(p_{1}, p_{2}, \ldots, p_{n}\right)$-balanced (quasi-balanced) if

$$
\lambda \bullet z \in D \quad \forall \lambda \in \overline{\mathbb{D}}, \forall z \in D,
$$

where for $z=\left(z_{1}, z_{2}, \ldots, z_{n}\right) \in D$, we define $\lambda \bullet z:=\left(\lambda^{p_{1}} z_{1}, \lambda^{p_{2}} z_{2}, \ldots, \lambda^{p_{n}} z_{n}\right)$. If $p_{1}=p_{2}=$ $\cdots=p_{n}=1$ above, then we say $D$ is a balanced domain (balanced domains are also known as complete circular domains in the literature).

Given a $\left(p_{1}, p_{2}, \ldots, p_{n}\right)$-balanced domain $D \subset \mathbb{C}^{n}$, we define the Minkowski function $\mathfrak{h}_{D}$ : $\mathbb{C}^{n} \rightarrow \mathbb{C}$ by

$$
\mathfrak{h}_{D}(z):=\inf \left\{t>0: \frac{1}{t} \bullet z \in D\right\}
$$

Clearly $D=\left\{z \in \mathbb{C}^{n}: \mathfrak{h}_{D}(z)<1\right\}$ and $\mathfrak{h}_{D}(\lambda \bullet z)=|\lambda| \mathfrak{h}_{D}(z)$. This function was first studied by Nikolov [Nik06] (see also [Bha06]). It turns out that $\mathfrak{h}_{D}$ is plurisubharmonic if and only if $D$ is additionally pseudoconvex; see [Bha06, Lemma 2.3].

Section 2.2 of [JP13] contains an extensive treatment of the properties of the Minkowski function of both balanced and quasi-balanced domains.

\subsection{The symmetric product of a Riemann surface}

Let $X$ be a Riemann surface. Given $\left(x_{1}, \ldots, x_{n}\right) \in X^{n}$, we denote by $\left\langle x_{1}, \ldots, x_{n}\right\rangle$ the image in the quotient topological space $\operatorname{Sym}^{n}(X):=X^{n} / S_{n}$ under the $S_{n}$-action on $X^{n}$ that permutes the entries of $\left(x_{1}, \ldots, x_{n}\right)$. We will also abbreviate the element

$$
\langle\underbrace{z_{1}, \ldots, z_{1}}_{\mu_{1} \text {-times }}, \underbrace{z_{2}, \ldots, z_{2}}_{\mu_{2} \text {-times }}, \ldots, \underbrace{z_{k}, \ldots, z_{k}}_{\mu_{k} \text {-times }}\rangle, \mu_{1}+\cdots+\mu_{k}=n,
$$


by

$$
\left\langle z_{1} ; \mu_{1}, \ldots, z_{k} ; \mu_{k}\right\rangle
$$

The map

$$
X^{n} \ni\left(x_{1}, \ldots, x_{n}\right) \longmapsto\left\langle x_{1}, \ldots, x_{n}\right\rangle \forall\left(x_{1}, \ldots, x_{n}\right) \in X^{n}
$$

will be denoted by $\pi_{\text {Sym }}^{n}$. We shall drop the superscript when there is no ambiguity. It is easy to see that there is a natural complex structure on $\operatorname{Sym}^{n}(X)$ that makes it a complex manifold of dimension $n$ (see below). With this complex structure, the map $\pi_{\text {Sym }}$ is a branched proper holomorphic mappings whose set of critical points is

$$
\left\{\left(z_{1}, \ldots, z_{n}\right) \in X^{n}: z_{i}=z_{j} \text { for some } i \neq j\right\} .
$$

The symmetrized polydisk $\mathbb{G}^{n}$ is a quasi-balanced domain in $\mathbb{C}^{n}$ with weights $(1,2, \ldots, n)$ defined using the elementary symmetric polynomials as follows. Let $\sigma_{j}, j=1, \ldots, n$, denote the elementary symmetric polynomial of degree $j$ in $n$ indeterminates. The map $\pi^{(n)}: \mathbb{C}^{n} \rightarrow$ $\mathbb{C}^{n}$ is defined as:

$$
\begin{array}{r}
\pi^{(n)}\left(z_{1}, \ldots, z_{n}\right):=\left(\sigma_{1}\left(z_{1}, \ldots, z_{n}\right), \sigma_{2}\left(z_{1}, \ldots, z_{n}\right), \ldots, \sigma_{n}\left(z_{1}, \ldots, z_{n}\right)\right), \\
\left(z_{1}, \ldots, z_{n}\right) \in \mathbb{C}^{n} .
\end{array}
$$

Again, we shall drop the superscript when there is no scope for confusion.

The symmetrized polydisk, $\mathbb{G}^{n}$, is defined as $\mathbb{G}^{n}:=\pi\left(\mathbb{D}^{n}\right)$. It is easy to see that $\mathbb{G}^{n}$ is a $(1,2, \ldots, n)$-balanced domain in $\mathbb{C}^{n}$, whence $\mathbb{G}^{n}$ is a holomorphic embedding of the $n$-fold symmetric product of $\mathbb{D}$ into $\mathbb{C}^{n}$. It is also easy to see that the Minkowski functional of $\mathbb{G}^{n}$ is given by

$$
\mathfrak{h}_{\mathbb{G}^{n}}\left(z_{1}, \ldots, z_{n}\right):=\max \left\{\left|\lambda_{1}\right|, \ldots,\left|\lambda_{n}\right|: \pi^{(n)}\left(\lambda_{1}, \ldots, \lambda_{n}\right)=\left(z_{1}, \ldots, z_{n}\right)\right\} .
$$

The above formula implies that $\mathbb{G}^{n}$ is pseudoconvex. This also follows by appealing to the fact that the proper image of a pseudoconvex domain is pseudoconvex. This automatically means that $\mathbb{G}^{n}$ is a domain of holomorphy.

We now give a brief description of the complex structure on the topological space $\operatorname{Sym}^{n}(X)$ when $X$ is Riemann surface. Given subsets $V_{j} \subseteq X$ that are open, let us write:

$$
\left\langle V_{1}, \ldots, V_{n}\right\rangle:=\left\{\left\langle x_{1}, \ldots x_{n}\right\rangle: x_{j} \in V_{j}, j=1, \ldots, n\right\}
$$

The set $\left\langle V_{1}, \ldots, V_{n}\right\rangle$ is an open subset of $X_{\text {sym }}^{n}$ by the defining property of the quotient topology. Given a point $p \in \operatorname{Sym}^{n}(X), p=\left\langle p_{1}, \ldots p_{n}\right\rangle$, choose a holomorphic chart $\left(U_{j}, \varphi_{j}\right)$ of $X$ at $p_{j}, j=1, \ldots, n$, such that

$$
U_{j} \cap U_{k}=\emptyset \text { if } p_{j} \neq p_{k} \quad \text { and } \quad U_{j}=U_{k} \text { if } p_{j}=p_{k} .
$$

The above choice of local charts ensures that the map $\Psi_{p}:\left\langle U_{1}, \ldots, U_{n}\right\rangle \rightarrow \mathbb{C}^{n}$ given by

$$
\Psi_{p}:\left\langle x_{1}, \ldots, x_{n}\right\rangle \longmapsto\left(\left(\varphi_{1}\left(x_{1}\right), \ldots, \varphi_{n}\left(x_{n}\right)\right), \ldots,\left(\varphi_{1}\left(x_{1}\right), \ldots, \varphi_{n}\left(x_{n}\right)\right)\right)
$$

is a homeomorphism. This follows from the Fundamental Theorem of Algebra. The collection of all such charts $\left(\left\langle U_{1}, \ldots, U_{n}\right\rangle, \Psi_{p}\right)$ produces a holomorphic atlas on $\operatorname{Sym}^{n}(X)$. The following lemma is easy to prove and we omit the proof. 
Lemma 22. Let $X$ be a Riemann surface and for $1 \leq k<n$, define

$$
V_{k}:=\left\{\left\langle z_{1}, \ldots, z_{n}\right\rangle \in \operatorname{Sym}^{n}(X): \text { the set }\left\{z_{1}, \ldots, z_{n}\right\} \text { has precisely } k \text { elements }\right\}
$$

Then $V_{k}$ is an analytic subvariety of $\operatorname{Sym}^{n}(X)$.

The book [JP13] contains an exhaustive account of the various properties of the symmetrized polydisk. The book [Whi72] is the canonical reference for the symmetric product in general.

\section{A Schwarz lemma for quasi-balanced domains}

The following version of Schwarz lemma for balanced domains is well-known. This version follows easily from the fact that holomorphic maps contract under the Lempert function and the relationship between the Lempert function and the Minkowski function of a balanced pseudoconvex domain.

Result 23 (Proposition 3.1.1 of [J]13]). Let $D_{1} \subset \mathbb{C}^{m}$ and $D_{2} \subset \mathbb{C}^{n}$ be balanced pseudoconvex domains with Minkowski functions $\mathfrak{h}_{1}$ and $\mathfrak{h}_{2}$, respectively. Then given any holomorphic map $f: D_{1} \rightarrow D_{2}$ with $f(0)=0$, we have

$$
\mathfrak{h}_{2}(f(z)) \leq \mathfrak{h}_{1}(z) .
$$

We will now prove an analogue of the above result for quasi-balanced domains.

Theorem 24. Let $D$ be $a\left(p_{1}, \ldots, p_{n}\right)$-balanced pseudoconvex domain with highest weight $p_{n}$. Then

$$
\mathfrak{h}_{D}^{p_{n}}(z) \leq \lambda_{D}(z, 0) \leq \mathfrak{h}_{D}(z) .
$$

Proof. First observe that the pseudoconvexity of $D$ ensures that $\log \mathfrak{h}_{D}$ is plurisubharmonic. Fix $0<\varepsilon<1$ and consider the set

$$
K:=\left\{w \in D: \mathfrak{h}_{D}(w)=\varepsilon\right\} .
$$

Note that $0 \notin \bar{K}$. Observe that for any $z \in D$ such that $0<\mathfrak{h}_{D}(z)<\varepsilon$, we can find $0<t<1$ such that for some $z^{\prime} \in K$, we have $t \bullet z^{\prime}=z$. Hence $\mathfrak{h}_{D}^{p_{n}}(z)=t^{p_{n}} \mathfrak{h}_{D}^{p_{n}}\left(z^{\prime}\right)$. As $0 \notin \bar{K}$, we can trivially write the inequality

$$
\mathfrak{h}_{D}^{p_{n}}(w) \leq C|w| \forall w \in K,
$$

for some $C>0$, suitably large. It is also easy to see that $|z| \geq t^{p_{n}}\left|z^{\prime}\right|$. Thus,

$$
\mathfrak{h}_{D}^{p_{n}}(z)=t^{p_{n}} \mathfrak{h}_{D}^{p_{n}}\left(z^{\prime}\right) \leq C t^{p_{n}}\left|z^{\prime}\right| \leq C|z|
$$

Therefore $\frac{\mathfrak{h}_{D}^{p_{n}}(z)}{|z|}$ is bounded in a neighbourhood of 0 . Thus, $\mathfrak{h}_{D}^{p_{n}} \in P_{D}(z, 0)$ whence $\mathfrak{h}^{p_{n}}(z, 0) \leq$ $\lambda_{D}(z, 0)$. This inequality is obviously also true if $\mathfrak{h}_{D}\left(z_{0}\right)=0$. 
Now for a fixed $z$ with $\mathfrak{h}_{D}(z) \neq 0$, consider the map

$$
\phi: \mathbb{D} \ni \lambda \mapsto \lambda \bullet \frac{z}{\mathfrak{h}_{D}(z)} \in D .
$$

We then have

$$
\lambda_{D}(z, 0) \leq \lambda_{\mathbb{D}}\left(\mathfrak{h}_{D}(z), 0\right)=\mathfrak{h}_{D}(z) .
$$

On the other hand, if $\mathfrak{h}_{D}(z)=0$, for each $n \in \mathbb{Z}_{+}$, the element $n \bullet z \in D$. We repeat the above argument with the element $n \bullet z$ instead of $\frac{z}{\mathfrak{h}_{D}(z)}$. It is clear that $\lambda_{D}(z, 0) \leq 1 / n$. This proves that $\lambda_{D}(z, 0) \leq \mathfrak{h}_{D}(z)$ and we are done.

The above theorem yields the following analogue of Schwarz lemma for pseudoconvex quasi-balanced domains.

Theorem 25 (Schwarz Lemma). Let $D_{1} \subset \mathbb{C}^{n}$ and $D_{2} \subset \mathbb{C}^{m}$ be pseudoconvex quasi-balanced domains. If $f: D_{1} \rightarrow D_{2}$ is holomorphic and $f(0)=0$ then

$$
\mathfrak{h}_{D_{2}}^{p}(f(z), 0) \leq \mathfrak{h}_{D}(z, 0) \forall z \in D,
$$

where $p$ is highest weight of the quasi-balanced domain $D_{2}$

Remark 26. The above theorem subsumes Result 23 See [Bha06, Theorem 1.6] for a proof of the above Schwarz lemma using the Lempert function instead of the extremal function.

The following is a version of Schwarz lemma that follows from Theorem 25 This result was proved by Ransford-Nokrane [NR01] in a formulation involving algebroid multifunctions.

Theorem 27. Let $f: \mathbb{D} \rightarrow \mathbb{G}^{n}$ be holomorphic with $f(0)=0$ and $f(z)=\pi\left(\lambda_{1}, \ldots, \lambda_{n}\right)$. Then

$$
\max \left\{\left|\lambda_{1}\right|, \ldots,\left|\lambda_{n}\right|\right\} \leq|z|^{1 / n}
$$

Remark 28. As alluded to in the introduction, the above observation was the impetus for this paper.

\section{Proofs of the main results}

Our strategy is to establish that the function $\mathcal{H}_{\mathcal{M}_{X}}^{n}\left(\pi_{\text {Sym }}^{-1}(z), \pi_{\text {Sym }}^{-1}\left(z_{0}\right)\right)$ (see (1)), is intimately related to the extremal function of $\operatorname{Sym}^{n}(X)$ via a function $h_{1}$ which we will define below.

Let $X$ be a Riemann surface and fix $z_{0} \in \operatorname{Sym}^{n}(X)$. Define the function $h_{1}: \operatorname{Sym}^{n}(X) \rightarrow$ $[0,1)$ by

$$
h_{1}(z):=\max \left(\max _{i} \prod_{j} \mathcal{M}_{X}\left(z_{i}, a_{j}\right), \max _{i} \prod_{j} \mathcal{M}_{X}\left(z_{j}, a_{i}\right)\right) \text {, }
$$

where $z_{0}=\left\langle a_{1}, \ldots, a_{n}\right\rangle$ is a fixed point and we have written $z$ as $\left\langle z_{1}, \ldots, z_{n}\right\rangle$. We also define the function $h: \operatorname{Sym}^{n}(X) \rightarrow[0,1)$ by

$$
h(z):=\mathcal{H}_{\mathcal{M}_{X}}\left(\pi_{\text {Sym }}^{-1}(z), \pi_{\text {Sym }}^{-1}\left(z_{0}\right)\right)=\mathcal{H}_{\mathcal{M}_{X}}\left(\left\{z_{1}, \ldots, z_{n}\right\},\left\{a_{1}, \ldots, a_{n}\right\}\right),
$$


where $\pi_{\text {Sym }}^{-1}(z)$ and $\pi_{\text {Sym }}^{-1}\left(z_{0}\right)$ are defined as in Theorem 1 Observe that from the very definitions, we have

$$
h^{n}(z) \leq h_{1}(z) \forall z \in \operatorname{Sym}^{n}(X) .
$$

Our proof of the main theorem hinges on the following theorem combined with Lemma 8 and the fact that $\lambda_{\mathbb{D}}\left(x, x_{0}\right)=\mathcal{M}_{\mathbb{D}}\left(x, x_{0}\right)$. We, once again, emphasize that the Riemann surface $X$ is arbitrary and in view of this, Remark 5 is pertinent in what follows.

Theorem 29. Let $V$ be the set of critical values of the map $\pi_{\text {Sym }}: X^{n} \rightarrow \operatorname{Sym}^{n}(X)$. For each $z_{0} \in \operatorname{Sym}^{n}(X) \backslash V$, defining $h_{1}$ as in (4), we have

$$
h_{1} \in P_{\mathrm{Sym}^{n}(X)}\left(z_{0}\right) .
$$

Proof. From the very definition, $h_{1}$ is continuous, $h_{1}\left(z_{0}\right)=0$ and $0 \leq h_{1}<1$. We first show that the function $\log h_{1}$ is plurisubharmonic on $\operatorname{Sym}^{n}(X)$. Fix $z \in \operatorname{Sym}^{n}(X) \backslash V, z=$ $\left\langle z_{1}, \ldots, z_{n}\right\rangle$. Let $(U, \psi)$ be a coordinate chart around $z$ such that $\psi(z)=0$. We can find an open set $B \subset U$ such that:

1. The map $\left.\psi\right|_{B}$ is a biholomorphism onto a ball $B(0, r)$,

2. We can find an inverse $\left(\widetilde{\pi}_{1}, \ldots, \widetilde{\pi}_{n}\right)$ of $\pi_{\text {Sym }}$ defined on $B$ such that $\widetilde{\pi}_{i}(z)=z_{i}$.

For $y \in B$, we can write

$$
h_{1}(y)=\max \left(\max _{i} \prod_{j} \mathcal{M}_{X}\left(\tilde{\pi}_{i}(y), a_{j}\right), \max _{i} \prod_{j} \mathcal{M}_{X}\left(\tilde{\pi}_{j}(y), a_{i}\right)\right) .
$$

Now Proposition 20, together with basic properties of plurisubharmonic functions, shows that $\log h_{1}$ is plurisubharmonic on $\operatorname{Sym}^{n}(X) \backslash V$. By Riemann's removable singularities theorem for plurisubharmonic functions ([Gun90, Theorem 3, p. 178]), the function $h_{1}$ extends to be a plurisubharmonic function on $\operatorname{Sym}^{n}(X)$.

It remains to show that the final condition in the definition of $P_{X}\left(z_{0}\right)$ is satisfied by $h_{1}$. Let $(U, \psi)$ be any coordinate chart around $z_{0}$ such that $\psi\left(z_{0}\right)=0$. Choose $B$ and $\tilde{\pi}_{i}$ as before. Let $D_{i} \subset X$ be open pairwise disjoint coordinate disks that contain $a_{i}$. By continuity, shrinking $B$ if necessary, we can assume $\left(\tilde{\pi}_{1}, \ldots, \widetilde{\pi}_{n}\right)(B) \subset D_{1} \times \cdots \times D_{n}$. By the distance decreasing property of the Möbius pseudodistance and Remark 15 , we now have

$$
\max _{i} \mathcal{M}_{D_{i}}\left(\tilde{\pi}_{i}(z), a_{i}\right) \leq \mathcal{M}_{B}\left(z, z_{0}\right) \quad \forall z \in B .
$$

From Remark 14. $\mathcal{M}_{B}\left(z, z_{0}\right)=\frac{|\psi(z)|}{r}$. The above equation, combined with the fact that $\mathcal{M}_{X} \leq$ $\mathcal{M}_{D_{i}}$, therefore shows

$$
\max _{i} \mathcal{M}_{X}\left(\widetilde{\pi}_{i}(z), a_{i}\right) \leq \frac{|\psi(z)|}{r} \forall z \in B .
$$

From the very definition of $h_{1}$, it is now follows that

$$
h_{1}(z) \leq \frac{|\psi(z)|}{r} \forall z \in B .
$$

The function $h_{1}$ satisfies all the conditions required for it to be an element of $P_{X}\left(z_{0}\right)$ and we are done. 
Remark 30. It is not hard to see that $\lambda_{X}\left(\cdot, z_{0}\right) \in P_{X}\left(z_{0}\right)$ (see [Kli85 Corollary 1.3]). Therefore, in the definition of $h_{1}$, we might as well have used the function $\lambda_{X}$ instead of the function $\mathcal{M}_{X}$ and the same proof mutatis mutandis would show that the modified function is in $P_{X}\left(z_{0}\right)$ as well.

The following corollary is immediate from (6) and Theorem 29

Corollary 31. For each $z_{0} \in \operatorname{Sym}^{n}(X) \backslash V$

$$
h^{n}(z) \leq h_{1}(z) \leq \lambda_{\operatorname{Sym}^{n}(X)}\left(z, z_{0}\right) \text {. }
$$

Before we come to the proof of Theorem 1 , we need one final lemma.

Lemma 32. With the same notation as Theorem 1, let $1 \leq k \leq n$ be the highest integer such that for some $x^{0} \in \mathbb{D}$, writing $f\left(x^{0}\right)=\left\langle x_{1}^{0}, \ldots, x_{n}^{0}\right\rangle$, the set $\left\{x_{1}^{0}, \ldots, x_{n}^{0}\right\}$ has $k$ elements. Then:

1. Except for $x$ in a discrete set $E \subset \mathbb{D}, f(x)=\left\langle x_{1}, \ldots, x_{n}\right\rangle$ also has the property that $\left\{x_{1}, \ldots, x_{n}\right\}$ has $k$ elements;

2. For each $x \in \mathbb{D} \backslash E$, we can find a disk $V_{x} \subset \mathbb{D} \backslash E$ centred at $x$, holomorphic maps $\widetilde{f}_{x, 1}, \ldots, \widetilde{f}_{x, k}: V_{x} \rightarrow X$ and positive integers $\mu_{1}, \ldots, \mu_{k}$ whose sum is $n$ such that

$$
f(y)=\left\langle\widetilde{f}_{x, 1}(y) ; \mu_{1}, \widetilde{f}_{x, 2}(y) ; \mu_{2}, \ldots, \widetilde{f}_{x, k}(y) ; \mu_{k}\right\rangle, \forall y \in V_{x} .
$$

Proof. Let $E \subset \mathbb{D}$ be the set of all elements each $x \in \mathbb{D}$ with the property that $f(x)=$ $\left\langle x_{1}, \ldots, x_{n}\right\rangle$ is such that $\left\{x_{1}, \ldots, x_{n}\right\}$ has fewer than $k$ elements. By Lemma 22 the collection of all points $w$ in $\operatorname{Sym}^{n}(X)$ with the property that, writing $w$ as $\left\langle w_{1}, \ldots, w_{n}\right\rangle$, the set $\left\{w_{1}, \ldots, w_{n}\right\}$ has fewer than $k$ elements is an analytic subvariety of $\operatorname{Sym}^{n}(X)$ (Lemma 22). If $E$ is an indiscrete set, it follows from the principle of analytic continuation that $E=\mathbb{D}$, a contradiction.

Now let $x \in \mathbb{D} \backslash E$ and $f(x)=\left\langle x_{1} ; \mu_{1}, \ldots x_{k} ; \mu_{k}\right\rangle, \mu_{1}+\cdots+\mu_{k}=n$. Let $U_{i} \subset X$ be pairwise disjoint coordinate disks centred at $x_{i}$. Then by continuity, we can find a disk $V_{x} \subset \mathbb{D} \backslash E$ centred at $x$ such that

$$
f\left(V_{x}\right) \subset\langle\underbrace{U_{1}, \ldots, U_{1}}_{\mu_{1} \text {-times }}, \ldots, \underbrace{U_{k}, \ldots, U_{k}}_{\mu_{k} \text {-times }}\rangle .
$$

As the $U_{i}$ are pairwise disjoint and for each $y \in V_{x}$ and writing $f(y)=\left\langle y_{1}, \ldots, y_{n}\right\rangle$, the cardinality of $\left\{y_{1}, \ldots, y_{n}\right\}$ is $k$, it is clear that we can define continuous maps $\widetilde{f}_{x, 1}, \ldots, \widetilde{f}_{x, k}$ : $V \rightarrow X$ such that

$$
f(y)=\left\langle\widetilde{f}_{x, 1}(y) ; \mu_{1}, \widetilde{f}_{x, 2}(y) ; \mu_{2}, \ldots, \widetilde{f}_{x, k}(y) ; \mu_{k}\right\rangle, \forall y \in V_{x} .
$$

The fact that the maps $\widetilde{f}_{x, 1}, \ldots, \widetilde{f}_{x, k}$ are holomorphic is a simple consequence of the way the complex structure on $\operatorname{Sym}^{n}(X)$ is defined. 


\section{Proof of Theorem 1}

Let $E$ and $k$ be as in Lemma 32 For $x \in \mathbb{D} \backslash E$, we can find a disk $V_{x}$ and holomorphic maps $\widetilde{f}_{x, 1}, \ldots, \widetilde{f}_{x, k}$ defined on $V_{x}$ satisfying the conclusion of Lemma 32 . We now define $\hat{f}$ on $V_{x}$ by

$$
V_{x} \ni y \mapsto \pi_{\text {Sym }}^{k}\left(\widetilde{f}_{x, 1}(y), \ldots, \widetilde{f}_{x, k}(y)\right) .
$$

The above definition yields a holomorphic map $\hat{f}: \mathbb{D} \backslash E \rightarrow \operatorname{Sym}^{k}(X)$. By Riemann's removable singularities theorem for subharmonic functions, $\lambda_{\mathbb{D} \backslash E} \equiv \lambda_{\mathbb{D}} \mid \mathbb{D} \backslash E$. Fix $y \in \mathbb{D} \backslash E$ and define the functions $\hat{h}$ and $\hat{h}_{1}$ on $\operatorname{Sym}^{k}(X)$ with respect to the point $\hat{f}(y)$ and analogous to $h$ and $h_{1}$ (see (4) and (5)), respectively. It follows from Corollary 31 and Lemma 8 that

$$
\hat{h}^{n}(\hat{f}(x)) \leq \hat{h}_{1}(\hat{f}(x)) \leq \mathcal{M}_{\mathbb{D}}(x, y) \forall x \in \mathbb{D} \backslash E .
$$

It is obvious that

$$
h_{1}(f(x)) \leq \hat{h}_{1}(\hat{f}(x)),
$$

where $h_{1}$ is defined on $\operatorname{Sym}^{n}(X)$ with respect to the point $f(y)$. Thus

$$
h_{1}(x) \leq \mathcal{M}_{\mathbb{D}}(x, y) \forall x \in \mathbb{D} \backslash E .
$$

From (4), it is clear that if we view $h_{1}$ as a function of both $x$ and $y$, it is continuous on $\mathbb{D} \times \mathbb{D}$. So is the function $\mathcal{M}_{\mathbb{D}}(x, y)$. This combined with (6) delivers the theorem.

\section{Proof of Theorem 3}

In this proof, we shall tacitly identify $\operatorname{Sym}^{n}(\mathbb{D})$ with $\mathbb{G}^{n}$ without explicit mention. Let $z_{0}:=$ $f(0)=\left\langle a_{1}, \ldots, a_{n}\right\rangle$ and consider the functions $h$ as before defined on $\operatorname{Sym}^{n}(\mathbb{D})$ with respect to the point $f(0)$. It is harmless to assume that $0 \notin U$.

Claim: We can find an open disk $G \subset U$ and a holomorphic function $F: G \rightarrow \mathbb{D}$ such that for some $1 \leq j_{0} \leq n$, we have

$$
h^{n}(x)=\mathcal{M}_{\mathbb{D}}\left(F(x), a_{j_{0}}\right) \quad \forall x \in G .
$$

Proof of claim: We adopt the same notation as Lemma 32 Choose $x^{0} \in U \backslash E$. We have $h\left(x^{0}\right)=\mathcal{M}_{\mathbb{D}}\left(\widetilde{f}_{x^{0}, i_{0}}\left(x^{0}\right), a_{j_{0}}\right)$ for some choice of $1 \leq i_{0}, j_{0} \leq k$ (the choice might not be unique). Let $i_{0}, \ldots, i_{l}$ and $j_{0}, \ldots, j_{l}$ be all the indices such that $h\left(x^{0}\right)=\mathcal{M}_{\mathbb{D}}\left(\widetilde{f}_{x^{0}, i_{m}}\left(x^{0}\right), a_{j_{m}}\right)$ where $0 \leq m \leq l$. We can find a disk $G \subset V_{x^{0}} \cap U$ centered at $x^{0}$ such that for each $x \in G, h(f(x))$ is one of the functions $\mathcal{M}_{\mathbb{D}}\left(\widetilde{f}_{x^{0}, i_{m}}(x), a_{j_{m}}\right), 1 \leq m \leq l$. Define the sets

$$
E_{m}:=\left\{x \in G: h(f(x))=\mathcal{M}_{\mathbb{D}}\left(\widetilde{f}_{x^{0}, i_{m}}(x), a_{j_{m}}\right)\right\}, 0 \leq m \leq l .
$$

Each $E_{m}$ is a closed subset of $G$ and $\bigcup_{m=0}^{l} E_{m}=G$. Consequently, one of the sets $E_{m}$ has non-empty interior and we can rename $G$ to be any disk contained in this $E_{m}$ and choose $F$ to be the corresponding $\widetilde{f}_{i_{m}}$. 
With the claim in hand, the proof of the theorem is not hard. Let $a_{j_{0}}, G$ and $F$ be as in the claim. We may assume that $a_{j_{0}} \notin G$. Let $\phi \in \operatorname{Aut}(\mathbb{D})$ be the automorphism that interchanges 0 and $a_{j_{0}}$. We have $\left.h^{n}\left(\phi_{\text {Sym }} \circ f(x)\right)=\mathcal{M}_{\mathbb{D}}(\phi \circ F(x), 0)\right)^{n}=|\phi \circ F(x)|^{n} \forall x \in G$. By hypothesis, this means that

$$
|\phi \circ F(x)|^{n}=|x| \forall x \in G .
$$

But any branch of $\sqrt[n]{\cdot}$ on $G$ satisfies the above equation as well proving that for some $\theta$, $e^{i \theta}(\phi \circ F)$ is just some branch of the $\sqrt[n]{ }$. Let $\Theta_{\text {Sym }}$ be the automorphism of $\mathbb{G}^{n}$ associated to rotation by $e^{i \theta}$. Replacing $f$ with $\Theta_{\text {Sym }} \circ \phi_{\text {Sym }} \circ f$, we may assume that $\left.f\right|_{G}$ lifts over $\pi$ to a map into $\mathbb{D}^{n}$, one of whose components is a branch of $\sqrt[n]{\bullet}$.

Writing $f$ as $\left(f_{1}, \ldots, f_{n}\right)$, consider the polynomial over $O(\mathbb{D})$

$$
P(x, y):=y^{n}+f_{1}(x) y^{n-1}+\cdots+f_{n-1}(x) y^{n-1}+f_{n}(x)
$$

From the conclusion of the preceding paragraph, we can find a $n$-th root of unity $\zeta$ such that $P\left(x^{n}, x \zeta\right) \equiv 0$ on $G$. Consequently, $P\left(x^{n}, x \zeta\right) \equiv 0$ on $\mathbb{D}$ by the identity theorem. If $\eta$ is any other $n$-th root of unity, we see that $P\left(x^{n}, x \eta \zeta\right) \equiv 0$ on $\mathbb{D}$. Therefore $f(x)=\pi\left(\zeta_{1}(x), \ldots, \zeta_{n}(x)\right)$ where $\sqrt[n]{x}=\left\{\zeta_{1}(x), \ldots, \zeta_{n}(x)\right\}$. The theorem is proved with $g:=e^{i \theta} \phi$.

\section{A Schwarz lemma for the spectral unit ball}

In this section, we sketch a proof of a Schwarz lemma for the spectral unit ball. This theorem was formulated and proved by Bharali [Bha07]. But as the ideas fit well with the main themes of this article, we felt it is worthwhile to sketch a slightly different proof here.

For $n \in \mathbb{Z}_{+}$, the spectral unit ball $\Omega_{n} \subset \mathbb{C}^{n^{2}}$ is the collection of all matrices $A \in M_{n}(\mathbb{C})$ ( $n \times n$ complex matrices) whose spectrum $\sigma(A)$ is contained in $\mathbb{D}$. We have the following

Proposition 33. The set $\Omega_{n}$ is an unbounded balanced pseudoconvex domain with Minkowski function given by the spectral radius $\rho$.

Proof. That $\Omega_{n}$ is balanced and that the spectral radius is the Minkowski function is easy to see from the definitions. We can define the holomorphic map $\Psi_{n}: M_{n}(\mathbb{C}) \rightarrow \mathbb{C}^{n}$ given by $M \mapsto \pi(\sigma(M))$. Observe that $\Psi_{n}^{-1}\left(\mathbb{G}^{n}\right)=\Omega_{n}$ which shows that $\Omega_{n}$ is a domain of holomorphy (from that fact that $\mathbb{G}^{n}$ is a domain of holomorphy and [Hö90, Theorem 2.5.14]). Pseudoconvexity of $\Omega_{n}$ now follows from the characterization of domains of holomorphy (see [Hö90 Section 2.6]).

Remark 34. The above proposition shows that $\left.\rho\right|_{\Omega_{n}}$ is plurisubharmonic (see [JP13, Appendix B.7.6]). This fact is usually proved in the literature using a theorem of Vesentini [Ves68].

Definition 35. Given $A \in M_{n}(\mathbb{C})$, we can write its minimal polynomial $\mathbf{M}_{A}$ as

$$
\mathbf{M}_{A}(t)=\sum_{\lambda \in \sigma(A)}(t-\lambda)^{m(\lambda)} .
$$

The minimal Blaschke product corresponding to $A$ is defined by

$$
\mathbf{B}_{A}(t):=\prod_{\lambda \in \sigma(A) \subset \mathbb{D}}\left(\frac{t-\lambda}{1-\bar{\lambda} t}\right)^{m(\lambda)} .
$$


Using the minimal Blaschke product corresponding to $A$, we can construct a holomorphic map $\widetilde{A}: \Omega_{n} \rightarrow \Omega_{n}$ that takes $A$ to 0 . We define

$$
\widetilde{A}: B \mapsto \prod_{\lambda \in \sigma(A)}(\mathbb{I}-\bar{\lambda} B)^{-m(\lambda)}(B-\lambda \mathbb{I})^{m(\lambda)}
$$

where $m(\lambda)$ is the multiplicity of the eigenvalue $\lambda$ in the minimal polynomial of $A$. It can be shown that if $\sigma(B)=\left\{\lambda_{1}, \ldots, \lambda_{n}\right\}$ then $\sigma(\widetilde{A}(B))=\left\{\mathbf{B}_{A}\left(\lambda_{1}\right), \ldots, \mathbf{B}_{A}\left(\lambda_{n}\right)\right\}$. If $F: \mathbb{D} \rightarrow \Omega_{n}$ is holomorphic such that $F(z)=A$ and $F(w)=B$ then $\widetilde{A} \circ F$ takes $A$ to 0 and $\widetilde{B} \circ F$ takes $B$ to 0 . The following result is immediate from the Schwarz lemma for balanced domains (Result 23).

Result 36 (Bharali, Theorem 1.5 of [Bha07]). Let $f: \mathbb{D} \rightarrow \Omega_{n}$ be holomorphic. Then for $z, w \in \mathbb{D}$, we have

$$
\begin{array}{r}
\max \left\{\max _{\lambda \in \sigma(f(w))} \prod_{\mu \in \sigma(f(z))} \mathcal{M}_{\mathbb{D}}(\mu, \lambda)^{m(\mu)}, \max _{\mu \in \sigma(f(z))} \prod_{\lambda \in \sigma(f(w))} \mathcal{M}_{\mathbb{D}}(\mu, \lambda)^{m(\lambda)}\right\} \\
\leq \mathcal{M}_{\mathbb{D}}(z, w),
\end{array}
$$

where $m(\mu)$ and $m(\lambda)$ denote the multiplicity of the eigenvalues $\mu$ and $\lambda$ in $\mathbf{M}_{f(z)}$ and $\mathbf{M}_{f(B)}$, respectively.

\section{References}

[ALY13] Jim Agler, Zinaida A. Lykova, and N. J. Young, Extremal holomorphic maps and the symmetrized bidisc, Proc. Lond. Math. Soc. (3) 106 (2013), no. 4, 781-818.

[AY01] J. Agler and N. J. Young, A Schwarz lemma for the symmetrized bidisc, Bull. London Math. Soc. 33 (2001), no. 2, 175-186.

[BBDJ18] Gautam Bharali, Indranil Biswas, Divakaran Divakaran, and Jaikrishnan Janardhanan, Proper holomorphic mappings onto symmetric products of a Riemann surface., Doc. Math. 23 (2018), 1291-1311.

[Bha06] Gautam Bharali, Nonisotropically balanced domains, Lempert function estimates, and the spectral Nevanlinna-Pick problem, arXiv preprint math/0601107 (2006).

[Bha07]__ Some new observations on interpolation in the spectral unit ball, Integral Equations Operator Theory 59 (2007), no. 3, 329-343.

[CG15] Debraj Chakrabarti and Sushil Gorai, Function theory and holomorphic maps on symmetric products of planar domains, J. Geom. Anal. 25 (2015), no. 4, 2196-2225.

[CG18] Debraj Chakrabarti and Christopher Grow, Proper holomorphic self-maps of symmetric powers of balls, Arch. Math. (Basel) 110 (2018), no. 1, 45-52. 
[Cha17] Vikramjeet Singh Chandel, The 3-point spectral pick interpolation problem and an application to holomorphic correspondences, arXiv preprint arXiv:1710.02955, J. Geom. Anal. (to appear) (2017).

[Dem87] Jean-Pierre Demailly, Mesures de Monge-Ampère et mesures pluriharmoniques, Math. Z. 194 (1987), no. 4, 519-564.

[EZ05] Armen Edigarian and Wł odzimierz Zwonek, Geometry of the symmetrized polydisc, Arch. Math. (Basel) 84 (2005), no. 4, 364-374.

[Gun90] Robert C. Gunning, Introduction to holomorphic functions of several variables. Vol. I, The Wadsworth \& Brooks/Cole Mathematics Series, Wadsworth \& Brooks/Cole Advanced Books \& Software, Pacific Grove, CA, 1990, Function theory.

[HJ18] Pranav Haridas and Jaikrishnan Janardhanan, A note on the smoothness of the Minkowski function, arXiv preprint arXiv:1805.11023 (2018).

[Hö90] Lars Hörmander, An introduction to complex analysis in several variables, third ed., North-Holland Mathematical Library, vol. 7, North-Holland Publishing Co., Amsterdam, 1990.

[JP13] Marek Jarnicki and Peter Pflug, Invariant distances and metrics in complex analysis. 2nd extended ed., 2nd extended ed. ed., Berlin: Walter de Gruyter, 2013.

[Kli85] M. Klimek, Extremal plurisubharmonic functions and invariant pseudodistances, Bull. Soc. Math. France 113 (1985), no. 2, 231-240.

[Kli91] Maciej Klimek, Pluripotential theory, London Mathematical Society Monographs. New Series, vol. 6, The Clarendon Press, Oxford University Press, New York, 1991, Oxford Science Publications.

[Kob98] Shoshichi Kobayashi, Hyperbolic complex spaces, Grundlehren der Mathematischen Wissenschaften, vol. 318, Springer-Verlag, Berlin, 1998.

[Lem81] László Lempert, La métrique de Kobayashi et la représentation des domaines sur la boule, Bull. Soc. Math. France 109 (1981), no. 4, 427-474.

[Nik06] Nikolai Nikolov, The symmetrized polydisc cannot be exhausted by domains biholomorphic to convex domains, Ann. Polon. Math. 88 (2006), no. 3, 279-283.

[NR01] Abdelkrim Nokrane and Thomas Ransford, Schwarz's lemma for algebroid multifunctions, Complex Variables Theory Appl. 45 (2001), no. 2, 183-196.

[Sib81] Nessim Sibony, A class of hyperbolic manifolds, Recent developments in several complex variables (Proc. Conf., Princeton Univ., Princeton, N. J., 1979), Ann. of Math. Stud., vol. 100, Princeton Univ. Press, Princeton, N.J., 1981, pp. 357-372.

[Sta75] Charles M. Stanton, Bounded analytic functions on a class of open Riemann surfaces, Pacific J. Math. 59 (1975), no. 2, 557-565. 
[Ves68] Edoardo Vesentini, On the subharmonicity of the spectral radius, Boll. Un. Mat. Ital. (4) 1 (1968), 427-429.

[Whi72] Hassler Whitney, Complex analytic varieties, Addison-Wesley Publishing Co., Reading, Mass.-London-Don Mills, Ont., 1972.

[Zwo18] Włodzimierz Zwonek, Function theoretic properties of symmetric powers of complex manifolds, arXiv preprint arXiv:1804.09600 (2018). 